\title{
Expériences des immigrantes d'Asie du Sud en matière de respect dans les milieux de traitement du cancer
}

par Savitri Singh-Carlson, Anne Neufeld et Joanne Olson

\begin{abstract}
Abrégé
Le but de cette enquête ethnographique ciblée était d'examiner les expériences et perceptions de respect chez les immigrantes d'Asie du Sud dans le cadre des relations professionnels de la santé-clients et ce, dans le contexte d'une clinique canadienne de soins externes. Les caractéristiques du respect telles que décrites par les 11 femmes interviewées étaient la signification du respect, la façon d'être des professionnels de la santé, la façon dont ils s'occupent de la personne et la façon dont ils s'expriment oralement. La langue, les valeurs culturelles et les croyances ainsi que les facteurs sous-jacents d'ordre social, individuel et institutionnel qui coexistent avec l'aptitude des professionnels de la santé à créer le respect, constituaient quelquesunes des dimensions qui influençaient l'expérience du respect chez ces immigrantes. La capacité des professionnels de la santé à voir dans les immigrantes d'Asie du Sud des individus favorisait la formulation/construction du respect pour leur identité individuelle. Le besoin que l'on respecte " mon identité sociale » en tant qu'immigrante atteinte de cancer imprégnait les récits des femmes et ressortait de leurs expériences et perspectives personnelles.
\end{abstract}

Au Canada, un virage démographique a entrainé une hausse importante de l'immigration en provenance de pays non européens pendant les deux dernières décennies (Statistiques Canada, 2007). Les immigrantes, les refugiées et les femmes d'horizons ethniques variés constituent un pourcentage toujours croissant de la population canadienne (Statistiques Canada, 2007). L'hétérogénéité résultante de la population pose des défis en matière de prestation des soins aux personnes atteintes de maladies chroniques et de maladies mettant la vie en danger comme le cancer (Kirkham, 2003).

Selon les taux d'incidence actuels, 39 \% des femmes développeront un cancer durant leur vie (Société canadienne du cancer, 2008). En 2004, en moyenne, 1319 Canadiennes recevaient un diagnostic de cancer et 608 femmes décédaient de cette maladie chaque semaine (Société canadienne du cancer, 2008). Une comparaison des profils des femmes indiennes vivant en Inde, d'une part, et au Canada, d'autre part, a révélé de hauts taux d'incidence du cancer du sein chez ces deux groupes, mais des taux d'incidence plus élevés du cancer du col utérin chez les Indo-Canadiennes de ColombieBritannique (C.-B.) par rapport à l'ensemble des femmes de la province (Hislop et coll., 2007).

\section{Au sujet des auteures \\ Savitri Singh-Carlson, B.Sc.inf., Ph.D., Professeure adjointe, California State University Long Beach, 1250 Bellflower Boulevard, Long Beach, Californie, É-U. 90803. Tél. : 562230 1016; Téléc. : 562985 2382; Courriel : scarlson@csulb.edu \\ Anne Neufeld, inf., Ph.D., Professeure, Faculté des sciences infirmières, Université de l'Alberta, Edmonton, $A B$. \\ Joanne Olson, inf., Ph.D., Professeure et doyenne associée, Undergraduate Programs, Faculté des sciences infirmières Université de l'Alberta, Edmonton, $A B$.}

Les auteures souhaitent remercier toutes les participantes ainsi que les infirmières en oncologie qui ont contribué à la réalisation de cette recherche.

\section{Contexte}

Malgré la profusion dans la littérature de discussions sur le respect en tant que concept éthique et moral (Benner, 2002; Milton, 2008; Tarlier, 2004) et de composante fondamentale de la prestation des soins dans le contexte de la pratique (Barbosa \& Silva, 2007; Erlen, 1998), peu d'études ont réalisé un examen critique de la manière dont le respect est véhiculé au sein de la relation infirmière-client (Browne, 1995, 1997; Kelly, 1990). Encore moins d'études se sont penchées sur l'expérience du respect depuis la perspective des clients au sein des relations professionnels de la santé-clients dans les contextes de soins, particulièrement dans le cas des immigrantes. Une bonne compréhension de la gamme des facteurs sociaux, historiques, individuels et politiques qui sous-tendent les interactions de soins est essentielle si l'on veut que les prestataires fournissent des soins empreints de respect; en effet, ceci empêche la discrimination et la déshumanisation lesquelles marginalisent les patients aux horizons culturels variés lorsqu'ils accèdent au système de soins de santé conventionnel (Browne, 1995, 1997).

Des relations respectueuses réduisent les différences entre les individus et façonnent la manière dont les gens interagissent (Gallagher, 2007). Une récente étude auprès de d'immigrantes au Canada en provenance d'Asie du Sud indiquait que l'expérience initiale du diagnostic de cancer du sein est complexe puisqu'elle met en jeu des processus cognitifs individuels et des négociations avec autrui, notamment avec des professionnels de la santé (Bottorff, et coll., 2007). Quoique la plupart des femmes originaires du Canada sont confrontées aux divers défis associés au cancer, ces défis prennent habituellement une ampleur accrue chez les immigrantes qui font peut-être face à des enjeux de migration et d'établissement, notamment aux défis associés au maintien de leurs propres croyances et pratiques tout en apprenant de nouvelles façons de se donner une vie stable dans leur nouveau pays (Gupta, Kumar \& Stewart, 2002; Hilton et coll., 2001). Par exemple, les immigrantes doivent composer non seulement avec le diagnostic de cancer mais encore avec des enjeux liés à leur établissement au Canada tels que le fait d'avoir laissé leur famille étendue dans leur pays d'origine ou de prendre soin de membres de la famille et de s'adapter à la vie au Canada (Hilton et coll., 2001; Clegg, 2003; Singh, 2002).

Les disciplines du domaine de la santé comme les soins infirmiers, la médecine, le travail social et les professions liées au conseil font toutes du respect un principe éthique central qui oriente la pratique clinique (Beauchamp \& Childress, 1994; Morris, 1997). Bien que le fondement, les principes et valeurs des soins infirmiers comme l'autonomie, la dignité et l'intégrité reposent tous sur le concept de respect (Klutgen, 1995; Widäng, Fridlund \& Mårtensson, 2007), ces concepts sont utilisés à titre de synonymes de respect dans la littérature afin d'expliquer la dimension éthique du respect (Peters, 1961; Rokeach, 1979). Le but de cette étude qualitative était d'examiner le respect, une composante positive des relations professionnels de la santé-clients qui joue un rôle majeur dans la promotion de la santé et du bien-être, d'une part, et dans l'obtention et le maintien d'un niveau de santé optimal par l'individu, quelque soit son appartenance ethnique ou son sexe, d'autre part (Acton et coll., 1997).

\section{But}

Le but de cette étude était d'examiner les expériences et perceptions en matière de respect d'immigrantes d'Asie du Sud dans le contexte des relations professionnels de la santé-clients lors du traitement de leur cancer. L'étude faisait appel à la participation de femmes qui se rendaient dans deux cliniques de soins de suivi dispensés en ambulatoire pour y recevoir des traitements pour leur cancer. 


\section{Méthodologie}

Une approche ethnographique (Roper \& Shapiro, 2000) éclairée par la perspective féministe de Dorothy E. Smith (1987) abordait les significations du respect aux yeux des immigrantes d'Asie du Sud dans le cadre de leurs relations avec les professionnels de la santé et ce, dans le contexte social que constitue la clinique de cancérologie. L'ethnographie, une approche naturaliste, convenait à cette étude du fait de son utilisation répandue dans le domaine de la recherche sur les soins de santé comme mode de compréhension des expériences des individus concernant la maladie, leurs croyances culturelles relatives à la santé et les modèles de comportement dans le contexte social de leur vie (Morse \& Field, 1995). Afin de fournir des descriptions approfondies ou des récits détaillés des expériences des participantes - ce qui constitue le but principal de cette approche qualitative - le travail sur le terrain de grande intensité incluait comme principales sources de données l'observation des participantes, des entrevues approfondies en face à face, des notes de terrain détaillées et la tenue de journaux. La rigueur scientifique de l'étude a été établie en assurant la légitimité, la vérifiabilité et la crédibilité de cette enquête qualitative. Notre capacité de réflexion durant la collecte et l'analyse des données a permis d'amenuiser les biais des chercheuses au niveau des résultats.

L'étude a été approuvée par les comités de révision déontologique conjoints de l'université et du centre de cancérologie. Le recrutement a été effectué dans deux cliniques de cancérologie locales par les infirmières-chefs et les radiothérapeutes qui fournissaient une trousse d'information aux femmes qui pouvaient communiquer directement avec l'équipe de recherche. Les critères d'inclusion comprenaient les suivants : être une immigrante d'Asie du Sud, avoir dix-huit ans ou plus, avoir reçu un diagnostic de cancer et subir actuellement des traitements ou passer des visites cliniques de suivi. Ces patientes n'étaient pas en phase palliative. Aux fins de cette étude, les immigrantes d'Asie du Sud étaient des femmes d'origine indienne qui avaient émigré au Canada de l'Inde, du Pakistan, du Sri Lanka ou des îles Fidji.

\section{Collecte et analyse des données}

Sur une période de deux semaines, nous avons observé au travail, de trois à quatre heures à chaque fois, des professionnels de la santé de chaque discipline soignant des clients atteints de cancer afin d'observer et de dégager les activités habituelles de la matinée et de l'après-midi dans les milieux de soins et ce, avant de réaliser les entrevues. Notre intention n'était pas d'évaluer les professionnels et les disciplines dans les relations qu'ils entretiennent avec les clients mais plutôt d'utiliser les observations des participantes comme point de départ de la compréhension des relations dans le plus vaste contexte qu'est le travail quotidien des professionnels de la santé. Les observations des soins de cancérologie dispensés ne se limitaient pas aux immigrantes d'Asie du Sud mais concernaient toutes les clients fréquentant la clinique. Des observations additionnelles de participantes ont été effectuées à divers moments afin de clarifier, confirmer et valider des données contextuelles.

Les entrevues en face à face qui duraient de trente à soixante minutes ont été réalisées par la première auteure au domicile des femmes ou dans tout autre endroit de leur choix. Les questions de l'entrevue ont été mises à l'essai auprès de deux immigrantes d'Asie du Sud qui ne pouvaient participer à l'étude parce qu'elles n'avaient pas le cancer mais composaient malgré tout avec d'autres maladies chroniques. Les révisions apportées aux questions-guides de l'entrevue semi-dirigée permettaient aux femmes d'exprimer leurs récits en toute liberté. Quoique notre but premier était de comprendre les expériences et perceptions positives des participantes en matière de respect, nous avons également inclus des questions concernant des situations de manque de respect parce que celles-ci pouvaient exister dans le cadre des relations de soins en tant que contrepartie des situations positives en matière de respect. Quatre femmes qui avaient décrit tout une gamme de perspectives sur le respect ont été contactées et interviewées une seconde fois afin de confirmer, valider et approfondir leurs témoignages et de clarifier des variations dans les données. Les questions de la seconde série d'entrevues ont été formulées en fonction de l'examen et de l'analyse des données tirées de la première série d'entrevues auprès des femmes. Cette stratégie nous a aidées à dégager de nouveaux aperçus qui ont permis d'explorer et de clarifier des thèmes et des aspects des expériences similaires et dissimilaires des participantes relativement au respect. Browne (1995, 1997) a constaté que le fait d'interviewer des sujets une seconde fois permet de confirmer et de valider les données et de mieux comprendre les différentes significations du respect chez les femmes. Des membres de la famille, notamment des maris et des filles, ont facilité le processus d'entrevue parce qu'ils étaient les soignants primaires. Un formulaire de consentement rédigé en anglais et sa traduction écrite en pendjabi ou en hindi ont été remis à chacune des participantes avant la tenue de l'entrevue.

Une analyse comparative constante (Morse \& Field, 1995) des données saisies a été effectuée afin de cerner les caractéristiques récurrentes, convergentes et contradictoires au niveau des expériences des femmes, les thèmes et les catégories préliminaires et enfin, de sélectionner les citations parmi les données des entrevues. Les entrevues ont fait l'objet d'un enregistrement sonore puis d'une interprétation verbale vers l'anglais, celle-ci étant enregistrée sur support sonore avant de passer à l'étape de la transcription. L'exactitude des transcriptions a été vérifiée ligne par ligne avant de lancer l'analyse. On a demandé à une traductrice compétente en hindi ou en pendjabi de transcrire et de traduire deux entrevues afin de s'assurer de l'exactitude de la traduction par souci de crédibilité. Une réflexivité et une réflexion constantes ont permis de réduire l'imposition de suppositions préconçues. Il y a eu saturation des données quand les mêmes thèmes ont continué de s'en dégager.

Les données tirées de l'observation des participants et les notes de terrain prises lors de cet exercice ont été analysées de concert avec les données et les notes de terrain relatives aux entrevues afin d'éclairer la compréhension du travail des professionnels de la santé et de fournir de l'information sur le contexte des milieux cliniques. Au fur et à mesure de la progression de l'analyse préliminaire des données, de nouvelles observations des participants ont été menées afin de clarifier, confirmer et valider les données liées aux contextes. Le codage initial des données a été effectué ligne par ligne en vue de cerner les idées/expériences importantes. Les données y compris les notes et documents de terrain ont été analysées et cataloguées en fonction des codes au moyen de NVivo (7), un logiciel permettant de comparer et de contraster des catégories. Les catégories particulières et les thèmes plus abstraits ont été constamment raffinés à mesure de la saisie de nouvelles données et de l'approfondissement de l'analyse. Les données se rapportant à des idées/expériences similaires ont été regroupées dans des catégories apparentées avant de faire l'objet d'une synthèse visant à dégager les thèmes importants décrivant les aspects clés des expériences relatives au respect des immigrantes d'Asie du Sud.

\section{Résultats}

Onze femmes d'ascendance indienne et originaires d'Inde, du Pakistan, du Sri Lanka et des îles Fidji résidaient au Canada depuis trois à trente et un ans. Elles parlaient les langues suivantes : anglais, hindi, ourdou, pendjabi ou tamoul. Les religions de ces femmes allaient du christianisme à l'hindouisme en passant par l'islam et le sikhisme. Elles avaient entre quarante et quatre-vingts ans, la majorité d'entre elles ayant plus de soixante ans. Leurs antécédents en éducation variaient d'aucune scolarité à des diplômes universitaires. La plupart des femmes avaient pris leur retraite ou n'avaient jamais occupé d'emploi. Toutes les femmes étaient mariées à l'exception de quatre veuves. Elles étaient atteintes de cancers cérébraux, de cancers du sein, du colon ou de la langue. 


\section{Signification du respect}

Les récits des femmes relatifs au respect qui leur était manifesté sont ancrés dans le contexte de leurs expériences précédentes en matière de soins de santé, dans leur compréhension du déroulement de la maladie et dans leurs attentes en matière de respect à la lumière de leurs origines et de leur cadre de référence pour les relations avec des professionnels. La plupart des femmes disaient que les manifestations personnelles des croyances, valeurs, suppositions et attentes relatives au respect entretenaient des rapports avec les structures culturelles et sociales. Les femmes comprenaient que la langue et les termes employés pour décrire le respect et ses attributs pourraient différer pour des raisons de culture et d'appartenance ethnique; cependant, les comportements ou les manières d'accorder du respect étaient perçus comme étant similaires par les femmes de toutes les appartenances ethniques. Les participantes utilisaient constamment le terme sud-asiatique « ijjat » pour désigner la notion de respect et elles en rapprochaient la signification des expériences de traitement respectueux qu'elles avaient vécues dans les relations professionnels de la santé-clients. Les réels efforts des professionnels de la santé visant à mettre le diagnostic de cancer au second rang derrière le besoin de comprendre leur ethnicité et leur aptitude à reconnaître et valoriser ces femmes en tant qu'êtres humains aidaient ces dernières à se sentir chez elles dans un environnement qui leur était étranger. Ceci s'est avéré être un principe fondamental et un attribut très important du respect (ijjat) et de la participation à des relations, un aspect intrinsèque de l'humanité et de la participation à des relations sociales :

Le respect signifie considérer les autres comme des personnes, comme des individus qu'il convient d'aborder avec amabilité et politesse. Si on accepte les autres peu importe ce qu'ils sont ou qui ils sont, on fait preuve de respect. La race de la personne n'a aucune importance. C'est notre façon de parler qui diffère, c.-̀̀-d. que nous disons "ijjat » ( respect » en pendjabi) alors que les Canadiennes disent « respect». (3)

Il y a respect quand une personne, quelle qu'elle soit, vient chez toi pour te rendre visite; il faut lui parler en toute politesse et gentillesse. Tu lui parles avec amour et tu reconnais sa présence, peu importe de qui il s'agit. (1)

Il y avait concordance entre la manière dont les immigrantes d'Asie du Sud traitaient les personnes pénétrant dans leur demeure et la manière dont elles envisageaient le traitement qui leur serait accordé par les professionnels de la santé de la clinique. Le besoin d'être respectées pour "mon identité sociale » d'immigrante sudasiatique atteinte de cancer s'exprime dans l'ensemble des récits des femmes et est influencé par leurs expériences de vie personnelles, leur culture, leur sexe et leur importance au sein de l'unité familiale où leur âge exige le respect. Leur état cancéreux faisait naître des sentiments de peur et de perte, particulièrement à la lumière des récentes expériences de migration et de transition de certaines des femmes et du fait que bien des membres de leur famille étendue se trouvaient à l'étranger.

Je suis seule ici depuis le décès de mon mari. Je demeure avec de la parenté. Je vais peut-être y retourner pour être avec mes enfants. (3)

\section{La façon d'être des professionnels de la santé}

La plupart des femmes ont déclaré que le fait d'être considérées comme des individus sans tenir compte de leur âge, de leur sexe, de leur appartenance ethnique ni de leur classe sociale les aidait à découvrir leur plein pouvoir alors qu'elles faisaient face au cancer. En traitant les femmes à titre d'égales et en reconnaissant leur « humanité partagée », les professionnels de la santé permettaient aux femmes d'avoir leur propre perspective et leur propre identité sociale et d'apporter une contribution holistique à la relation professionnels de la santé-clients. Pour les femmes, la possibilité de s'exprimer était importante, même si elles ne pouvaient parler l'anglais, tandis qu'elles discutaient d'une maladie mettant leur vie en danger et touchant leur féminité et leur perception en tant que femmes. Elles avaient une voix puisqu'elles se faisaient entendre :

Il n'y a pas de différence entre les femmes du Canada et les immigrantes ... seulement la langue; nous ne sommes pas capables de nous exprimer comme elles le font, c'est tout un défi. (5)

Ma langue maternelle, dans ma langue, je trouve qu'il m'est plus facile d'exprimer ce que je ressens et de me mériter le respect des gens. "Dans ma langue à moi, je pourrais leur montrer qui je suis ». (4)

Être acceptée et traitée d'égal à égal constituait un attribut tandis que l'accueil était perçu comme une composante du respect puisqu'il donnait aux femmes l'impression que c'est en partenariat avec les professionnels de la santé qu'elles traversaient l'épreuve du cancer. Les femmes disaient de l'accueil des professionnels de la santé qu'il était d'une authenticité telle qu'il dépassait la simple courtoisie, qu'il leur souhaitait la bienvenue au sein de la relation professionnel de la santé-cliente. Elles voyaient dans la façon d'accueillir autrui un principe fondamental du respect et de la participation aux relations ainsi qu'un aspect intrinsèque de la nature humaine et une attente liée à la participation aux relations sociales. Cette authenticité dans l'accueil était bien plus que de la courtoisie, de la politesse ou des bonnes manières; elle reconnaissait la femme au sein de la relation :

Cela dépend du comportement de la personne et de la manière dont elle te parle et ensuite de la manière dont elle te dispense des soins. Il lui faut d'abord manifester du respect envers cette personne en lui disant "bonjour ", en adoptant un comportement respectueux et poli, après, elle peut passer à la prestation des soins. (4)

\section{La façon de dispenser des soins des professionnels de la santé}

Selon les participantes, la pudeur et le souci de préserver son intimité sont probablement des attributs inhérents de la condition de femme; toutefois, l'étendue de cette timidité à l'égard du corps féminin peut être particulière à chaque femme en fonction de ses expériences passées et de ses antécédents culturels et ce, quel que soit son âge. La plupart des femmes estimaient que les professionnels de la santé préservaient leur humanité au sein de la relation professionnel de la santé-client en respectant leur féminité et leur vulnérabilité bien qu'elle ait lieu dans un milieu clinique. La majorité des femmes ont confié que cette timidité et cette pudeur faisaient partie intégrante de leur culture sud-asiatique du fait de leur non exposition aux tenues occidentales :

Mon besoin d'intimité peut différer de celui d'autres. Je veux que la porte soit fermée et les stores baissés; c'est ma façon d'être de femme du Sud asiatique. En préservant mon intimité, on manifeste du respect et de la considération pour ma féminité. Il en serait de même pour les femmes de tous les groupes ethniques. (3)

Les femmes sentaient qu'elles étaient essentiellement dignes de respect et d'attention lorsque les professionnels de la santé manifestaient leur réel souci pour autrui en retenant une approche ouverte qui ne permettait pas toujours aux suppositions concernant les choses à faire et à ne pas faire en vertu des tabous culturels d'empêcher la reconnaissance d'autrui, particulièrement lorsque les professionnels de la santé prenaient le temps de prier avec les femmes. Pour la majorité des femmes, les visites cliniques revêtaient une signification particulière parce qu'elles éprouvaient de profonds liens humains lorsque les professionnels de la santé prenaient le temps de leur fournir leur pleine attention et de les soigner au moment de leur vie où elles en avaient le plus grand besoin. Une femme qui subissait une radiothérapie crânienne, affirmait s'être sentie respectée lorsque la professionnelle de la santé l'a traitée de la manière dont elle traiterait une invitée dans son propre foyer. En 
lui remettant sa tuque après la radiothérapie, la professionnelle de la santé l'avait dignifiée et lui avait redonné toute sa plénitude. Ce récit montrait bien que les manifestations du respect n'avaient pas besoin d'être extravagantes; quelquefois, les petits détails faisaient toute la différence dans le degré de respect manifesté ou dans son absence :

Ils m'ont aidée à me placer sur la civière ... ils ont mis des vis sur le côté de ma tête, vous savez, pour la machine. Il faut que je sois sous la machine et j'enlève ma tuque. Ils me manifestaient du respect ... puis, ils m'ont aidée à mettre mon blouson et ma tuque. C'étaient là des comportements respectueux. (10)

\section{Façon de parler des professionnels de la santé}

Comme la plupart des femmes pensaient que les expériences de soins vécues dans un établissement clinique étaient généralement très invasives et touchaient la personne atteinte dans sa globalité, le fait de les accueillir " en disant " bonjour" ou en faisant un signe de la tête à cet effet » avant de dispenser les soins humanisait la relation. À l'opposé, certaines femmes se sentaient rabrouées quand des médecins les abordaient dans la plus grande indifférence en leur demandant « quel est donc le problème? » sans prendre le temps de dire " bonjour», pourtant une salutation universelle.

Les salutations impliquaient des comportements verbaux et non verbaux tels qu'un hochement de la tête, un sourire, un contact visuel ou le fait de dire " au revoir » à la fin, ce qui donnait aux femmes une place au sein de la relation. Les marques subtiles de respect telles que déterminées par l'apparence physique, l'attitude, le comportement ou les actions du professionnel de la santé dès le début de la relation donnaient le ton à une relation empreinte de respect. La plupart des femmes jugeaient que ces comportements formaient une petite partie du tout qui les encourageait à suivre leurs traitements quotidiens de radiothérapie lesquels peuvent s'étendre sur trente-cinq jours :

Elles me disent toujours "bonjour, comment allez-vous? ». Même après le traitement, elles m'aident à me rhabiller et me disent "au revoir, à demain ». J'étais heureuse et j'avais le cour en paix et je n'avais pas peur de revenir pour mon traitement suivant. (6)

Je crois que le respect se manifeste dans la manière dont elles sont élevées, dans leur attitude globale, dans leurs croyances, leurs valeurs et la façon dont elles considèrent les autres. Le respect imprègne leurs actions. (2)

La plupart des femmes ont indiqué que le style de communication et de fourniture d'information des professionnels de la santé les respectaient et aidaient à les mettre à l'aise alors qu'elles se sentaient vulnérables. Les femmes croyaient que tous les patients atteints de cancer sont dépassés par leur maladie et qu'ils doivent composer avec une foule de choses telles que leur mortalité, la possibilité de devoir laisser dans le deuil tous leurs proches, prendre des décisions relativement à leur emploi et à leur qualité de vie concernant les traitements et leurs effets secondaires. Une jeune femme de 40 ans qui avait un enfant se sentait respectée quand les professionnels de la santé faisaient consciemment des efforts pour engager le dialogue avec elle et quand ils lui fournissaient des explications sur l'état de son cancer et de précieux renseignements sur son organisme :

Quand tu apprends que tu as le cancer, ton cerveau est surmené, avec toutes ces pensées sur tout et rien, tu sais. Pourtant, je me sentais en toute sûreté ... ils m'ont expliqué l'information relative à mon cancer. Je n'avais pas peur; je me sentais libre. (7)

En revanche, d'autres femmes estimaient que la façon de parler et d'engager le dialogue de certains professionnels de la santé - des médecins pour la plupart - pouvait fermer la porte sur de nouvelles interactions ou au contraire l'ouvrir, particulièrement lorsqu'ils utilisaient une terminologie médicale complexe. Il n’y avait pas que la quantité et la qualité de l'information fournie qui rehaussaient les expériences de respect chez les femmes mais encore la qualité de la prestation et le style de communication :

Il parlait en utilisant ses termes médicaux à lui ... les mots justes. Parfois, tu crois qu'il ne veut pas faire attention à toi, mais c'est la terminologie qu'il utilise tout le temps; ce n'est pas sa personnalité. (8)

Étant donné que certaines femmes n'avaient pas l'occasion de parler anglais régulièrement, elles éprouvaient de la frustration quand elles avaient du mal à s'exprimer relativement à leurs expériences en matière de soins. Ce sentiment de frustration n'était pas dû à leur manque de maîtrise de la langue anglaise mais au fait qu'elles ne parvenaient pas à trouver les mots, termes ou expressions décrivant exactement ce qu'elles ressentaient. Elles en étaient marginalisées durant les interactions parce qu'il leur était impossible de parler du plus profond de leur être des souffrances et de la douleur associées à leur état cancéreux. Quelques femmes ont confié qu'il y avait des fois où elles ne voulaient pas utiliser l'anglais parce que les souffrances, la fatigue et la douleur leur dérobaient une part considérable de leur précieuse énergie dont elles avaient tant besoin pour effectuer les activités de la vie quotidienne. L'une d'elles a rapporté l'ampleur de l'effort qu'il lui fallait fournir pour se rappeler d'utiliser l'anglais lors des moments de souffrance :

D'habitude, je comprends l'anglais mais quand j'éprouve beaucoup de douleur, je ne veux pas parler anglais. Je ne veux pas parler/penser. Si on me parle lentement, j'arrive à comprendre. (1)

Lorsque les femmes avaient des interactions avec des interprètes, elles en étaient reconnaissantes et y trouvaient une marque de respect puisque les interprètes leur fournissaient des renseignements et des explications sur les traitements. Par contre, certains professionnels de la santé ignoraient que les immigrantes d'Asie du Sud appartiennent à différents groupes linguistiques. Les suppositions erronées des professionnels de la santé pouvaient entraver les efforts des patientes en vue de comprendre l'information. Par exemple, des interprètes parlant le pendjabi étaient fournies à des femmes de langue hindi :

Au début, ... il y avait une Indienne qui expliquait en hindi. Il y a, tu sais, des femmes pendjabi qui parlent l'hindi mais la plupart d'entre elles ne parlent que le pendjabi, et il est difficile de les comprendre. (9)

La plupart du temps, il y a des interprètes. Parfois, il n'y a pas d'interprète, mais ma belle-sour parle l'anglais [sa belle-sœur l'accompagne parfois pour jouer le rôle d'interprète, mais la majorité du temps, elle doit compter sur l'interprète fournie par l'hôpital]. Je ne parle peut-être pas l'anglais, mais je comprends le médecin. Je ne peux pas m'expliquer, alors je dois parler hindi à l'interprète. (11)

Ces femmes croyaient que quelques-uns des professionnels de la santé avaient des idées reçues qui aboutissaient à des stéréotypes sur les femmes lesquels réduisaient le respect et créaient des obstacles à la communication de l'information pertinente sur les traitements. Les femmes suggéraient aux professionnels de la santé d'adopter une approche plus individualisée et de demander aux femmes de préciser leur degré de maîtrise orale de l'anglais et ce, quel que soit l'âge ou l'appartenance ethnique des femmes. Les participantes suggéraient aux professionnels de la santé de demander dès le départ aux membres de la famille ou aux femmes quelle langue ils et elles parlent ou préfèrent et de fournir une interprète qui parle la langue de la patiente concernée. Étant donné que la plupart des patients d'origine sud-asiatique recevant des soins dans ces cliniques ont le pendjabi, l'hindi ou l'ourdou comme langue maternelle et l'anglais comme langue seconde, cette stratégie aiderait les patientes à améliorer leur compréhension du cancer. En outre, les femmes étaient d'avis que cette approche de la relation professionnel de la santé-client servirait à réduire les suppositions et les stéréotypes et à accroître le respect : 
L'interprète était une femme pendjabi d'Inde et elle s'adressait à moi en hindi. Certaines femmes pendjabi parlent très bien l'hindi, mais d'autres non. De plus, la plupart des femmes pendjabi ont tendance à parler d'une voix grave dans leur langue et il est difficile de les comprendre. À la clinique de chimiothérapie, il y a quelques infirmières pendjabi, mais l'une d'entre elles ne comprend pas l'hindi. (2)

Quoique la plupart des femmes préfèrent discuter de questions liées à l'appareil génital féminin avec des professionnelles de la santé, elles étaient résignées à l'idée de recevoir leurs soins de professionnels de la santé de l'un ou l'autre sexe. Cependant, bien des femmes ont rapporté que le fait de disposer de femmes interprètes qui parlaient leur langue était une source de réconfort car c'est par l'intermédiaire d'une femme interprète parlant leur langue qu'elles pouvaient s'adresser au professionnel de la santé masculin.

Le respect manifesté par les professionnels de la santé lors des visites cliniques faisait naître un sentiment de sécurité et d'aise chez les femmes et ce, quelle que soit l'ambiguïté de leur condition. La majorité des femmes se sentaient « comme chez elles » dans le milieu clinique grâce à la façon dont les professionnels de la santé leur parlaient et ce, indépendamment de la maîtrise de l'anglais oral chez les femmes. Savoir manifester du respect envers autrui en reconnaissant sa présence et en saluant autrui quels que soient sa position sociale, sa religion, son genre, son âge, sa culture ou son état de santé est une qualité inhérente qui valorise l'identité sociale d'autrui.

\section{Discussion}

Le thème central « l'individu que je suis » est conforme aux récits des femmes souhaitant que l'on reconnaisse en elles des individus ayant une identité sociale; un point de vue. La complexité de la prestation des soins à des clients aux horizons culturels variés peut être abordée en créant des environnements sûrs permettant la tenue de discussions franches mais respectueuses au sein des relations professionnels de la santé-clients. L'étude de Browne (1995) auprès d'une population autochtone vient confirmer les résultats selon lesquels un rapport entre les attitudes et le comportement des prestataires de soins envers les femmes autochtones et l'incidence d'interactions cliniques respectueuses créaient des sentiments d'égalité et de dignité. Clegg (2003) a signalé que le concept de respect pour un individu était influencé en termes globaux par des interprétations politiques, sociales, économiques et religieuses.

Dans leur étude - réalisée auprès d'immigrantes d'Asie du Sud sur la «stigmatisation de l'autre » dans les services de santé, Johnson et ses collaborateurs (2004) ont souligné la nécessité d'avoir dans le milieu de soins des valeurs telles que l'égalité et le respect à l'égard de la diversité; cependant, ces notions peuvent parfois être utilisées de façons qui occultent les barrières et désavantages bien concrets auxquels sont confrontés la plupart des immigrants sur le plan de l'accès aux soins de santé et sur l'adaptation à la vie au Canada. Vydelingum (2006) constatait qu'en ne voyant que les différences dans les « autres » cultures, les professionnels de la santé avaient tendance à adopter une approche réductionniste en vertu de laquelle les infirmières appliquaient aux immigrants « un ensemble de choses à faire et de choses à ne pas faire » (p. 29, traduction libre) plutôt que de voir en elles des individus ayant chacun leur identité propre.

La fourniture de soins adaptés aux différences culturelles nous amène à remettre en question nos propres suppositions relatives aux groupes culturels auxquels appartiennent les clients que nous soignons, à situer les préoccupations de santé des femmes dans un cadre structurel et à relier la dynamique des consultations individuelles à de plus vastes enjeux sociaux, politiques et économiques (Browne, 2003; Harris \& Templeton, 2001; Spitzer et coll., 2003). Hilton et ses collègues (2001), dans leur étude concernant des immigrantes d'Asie du Sud au Canada, ont confirmé qu'en respectant le besoin que ressentaient ces femmes de poursuivre l'utilisation des remèdes traditionnels de leur région d'origine, on aidait à « maintenir et préserver leur patrimoine et identité culturels de femme du Sud asiatique »(p. 558, trad. libre). Bottorff, Johnson et leurs collègues (1998), dans une étude portant sur les croyances liées au cancer du sein de femmes d'Asie du Sud, insistaient qu'il était nécessaire de respecter et de reconnaître les liens entre les croyances des femmes relatives à leur vocation dans la vie et à leur pudeur, d'une part, et la manière dont elles se présentent dans la société et les rôles qu'elles doivent jouer dans la société et dans la collectivité, d'autre part.

Les résultats de la présente étude révèlent que la capacité des professionnels de la santé à faire montre de respect en saluant sincèrement et spontanément les femmes et en les traitant à titre d'individus dans les interactions dès le début de la relation professionnel de la santé-cliente aidait à lui conférer une approche « axée sur la personne », où l'identité primait sur l'ethnicité. Des résultats similaires mettaient en relief la nature ouverte de la communication et un réel intérêt envers la personne en reconnaissant sa présence au commencement de la consultation comme élément de la construction d'une relation de soins professionnelle et empreinte de bienveillance au sein de laquelle les patients sentent qu'on les traite comme des personnes considérées dans leur globalité dont les besoins psychosociaux, physiques et émotionnels ont été satisfaits (Liu, Mok \& Wong, 2005; Thorne et coll., 2005).

La présente étude a révélé un manque de perception de la part des professionnels de la santé et de la direction de la clinique relativement à l'éventail des langues parlées par les femmes originaires d'Asie du Sud. De même, il sera peut-être nécessaire d'examiner attentivement les politiques et directives régissant les procédures des services d'interprétation fournis par la clinique afin de sensibiliser le personnel à l'existence de divers sous-groupes au sein de la communauté sud-asiatique dont les membres parlent différentes langues. Anderson (1998) a indiqué qu'il fallait remettre en question les politiques de santé actuelles concernant les services d'interprétation et la documentation publiée dans des langues étrangères pour des populations diverses. Fitch et ses collègues (1998) rapportaient que les infirmières en oncologie doivent relever divers défis dans les milieux cliniques lorsqu'elles dispensent des soins et administrent des traitements complexes contre le cancer et que ces défis étaient rehaussés lorsque les infirmières œuvraient auprès d'immigrants ne maîtrisant pas nécessairement l'anglais. Les résultats d'autres études ont conclu que dans les établissements desservant une importante population d'immigrants, les interprètes peuvent assumer les rôles de médiateurs culturels et de défenseurs des intérêts des patients dans le contexte de la cancérologie, ce qui se solderait par une réduction du stress et de l'épuisement émotionnels et une amélioration des relations de soins, particulièrement s'ils font partie du personnel de l'établissement puisqu'ils ont ainsi une bonne connaissance de ses politiques et procédures en matière de soins (Singh, 2002; Vydelingum, 2005).

La promotion de l'idée selon laquelle le patient est un « autre » être humain au sein de la relation peut être réalisée en favorisant chez les professionnels de la santé la conscience de soi critique relativement à leur culture et à la reconnaissance de ses préjugés, deux exercices qui peuvent être appris et encouragés afin d'empêcher de stéréotyper les « autres ». De plus, l'adaptation du style de communication au patient individuel, au type de cancer et à ses traitements afférents se soldera par la création de relations respectueuses et satisfaisantes à la fois pour les patients et pour les professionnels de la santé (Bottorff et coll., 2007).

\section{Conclusion}

Comme cette étude portait uniquement sur les immigrantes d'Asie du Sud, il est possible que les résultats ne s'appliquent pas aux femmes d'autres origines ethniques ni aux hommes. Une plus 
grande diversité des niveaux de revenus et de scolarité parmi les participantes et de plus importantes variations dans l'âge des femmes pourraient donner d'autres perspectives du respect. Dans une certaine mesure, le nombre relativement faible d'entrevues menées dans le cadre de cette étude pourrait constituer un facteur limitatif. Toutefois, la nature diversifiée des sous-groupes a permis d'accroître la représentativité de la population examinée. Cette étude a également tiré profit de la vaste gamme des expériences des participantes en matière de types de cancer et des observations de ces dernières sur la prestation des soins dispensés par les membres

\section{Références}

Acton, G.J., Irvin, B.L., Jensen, J A., Hopkins, B.A., \& Miller, E.W. (1997). Explicating middle-range theory through methodological diversity. Advances in Nursing Science, 19(3), 78-85.

Anderson, J.M. (1998). Speaking of illness: Issues of first generation Canadian women-Implications for patient education and counselling. Patient Education and Counselling, 33, 97-207.

Barbosa, I.A., \& Silva, M.J. (2007). Humanization in nursing care: Acting with respect in a school hospital. Revista Brasileira de Enfermagem, 60, 546-51.

Beauchamp, T.L., \& Childress, J.F. (1994). Principles of biomedical ethics (4th ed.). New York: Oxford University Press.

Benner, P. (2002). Creating compassionate institutions that foster agency and respect. American Journal of Critical Care, 11, 164-166.

Bottorff, J.L., Grewal, S., Balneaves, L.G., Naidu, P., Johnson, J.L., \& Sawhney, R. (2007). Punjabi women's stories of breast cancer symptoms. Cancer Nursing, 30(4), E36-E45.

Bottorff, J.L., Johnson, J.L., Bhagat, R., Grewal, S., Balneaves, L.G., Clarke, H., et al. (1998). Beliefs related to breast health practices: The perception of South Asian women living in Canada. Social Sciences \& Medicine, 47, 2075-2098.

Browne, A.J. (1995). The meaning of respect: A First Nation's perspective. Canadian Journal of Nursing Research, 27(4), 95-109.

Browne, A.J. (1997). A concept analysis of respect: Applying the hybrid model in cross-cultural settings. Western Journal of Nursing Research, 19, 762-780.

Browne, A.J. (2003). First Nations women and health care services: the socio-political context of encounters with nurses. Unpublished doctoral dissertation, University of British Columbia, Vancouver, BC, Canada.

Clegg, A. (2003). Older South Asian patient and carer perceptions of culturally sensitive care in a community hospital setting. Journal of Clinical Nursing, 12, 283-290.

Erlen, J.A. (1998). Culture, ethics, and respect: The bottom line is understanding. Orthopaedic Nursing, 17(6), 79-82.

Fitch, M.I., Greenberg, M., Cava, M., Spaner, D., \& Taylor, K. (1998). Exploring the barriers to cervical screening in an urban Canadian setting. Cancer Nursing, 21, 441-449.

Gallagher, A. (2007). The respectful nurse. Nursing Ethics, 14, 360-371.

Gupta A., Kumar A., \& Stewart, D.E. (2002). Cervical cancer screening among South Asian women in Canada: The role of education and acculturation. Health Care for Women International, 23, 123-134.

Harris, S.R., \& Templeton E. (2001). Who's listening? Experiences of women with breast cancer in communicating with physicians. Breast Journal, 7, 444-449.

Hilton, B.A., Grewal, S., Popatia, N., Bottorrf, J.L., Johnson J.L., Clarke, H., et al. (2001). The desi ways: Traditional health practices of South Asian women in Canada. Health Care for Women International, 22, 553-567.

Hislop, T.G., Bajdik, C.D., Saroa. S.R., Yeole B.B., \& Barroetavena M.C. (2007). Cancer incidence in India from three areas: Delhi and Mumbai, India, and British Columbia, Canada. Journal of Immigrant and Minority Health, 9, 221-227. des diverses disciplines œuvrant dans le contexte clinique. De futurs travaux pourraient examiner les perceptions de respect des femmes dans les relations mettant en jeu des infirmières, d'une part, et d'autres professionnels de la santé des cliniques, d'autre part. Les résultats de la présente recherche suggèrent qu'il vaudrait la peine de mener d'autres travaux sur les populations vulnérables et les styles de communication des professionnels de la santé dans le cadre de la prestation de soins liés au cancer, particulièrement lorsque la communication étaye le partenariat soignant-client dans les soins de cancérologie.

Johnson, J.L., Bottorff, J.L., Browne, A.J., Grewal, S., Hilton, B.A., \& Clarke, H. (2004). Othering and being othered in the context of health care services. Health Communication, 16, 253-271.

Kelly, B. (1990). Respect and caring: Ethics and the essence of nursing. In M.M. Leininger (Ed.), Ethical and moral dimensions of care (pp. 213-221). Detroit, MI: Wayne State University Press.

Kirkham, S. (2003). The politics of belonging and intercultural health care. Western Journal of Nursing Research, 25, 762-780.

Klutgen, J. (1995). Autonomy and intervention. New York: Oxford University Press.

Liu, J.E., Mok, E., \& Wong, T. (2005). Perceptions of supportive communication in Chinese patients with cancer: Experiences and expectations. Journal of Advanced Nursing, 52, 262-270.

Milton, C.L., (2008). Boundaries: Ethical implications for what it means to be therapeutic in the nurse-person relationship. Nursing Science Quarterly, 21, 18-21.

Morris, N.M. (1997). Respect: Its meaning and measurement as an element of patient care. Journal of Public Health Policy, 18, 133-151.

Morse, J.M., \& Field, P.A. (1995). Qualitative research methods for health professionals (2nd ed.). Thousand Oaks, CA: Sage.

Peters, R.S. (1961). Respect for persons and fraternity. In R.S. Peters (Ed.), Ethics and education (pp. 133-137). Palo Alto, CA: Scott \& Foresman.

Rokeach, M. (1979). Understanding human values. New York: Free Press.

Roper, J.M., \& Shapiro, J. (2000). Ethnography in nursing research. Thousand Oaks, CA: Sage.

Singh, S.W. (2002). An exploration of South Asian women's experiences following abnormal pap smear results. Unpublished master's thesis, University of British Columbia, Vancouver, BC, Canada.

Smith, D.E. (1987).The everyday world as problematic: A feminist sociology. Boston: North Eastern University Press.

Société canadienne du cancer (2008). Statistiques canadiennes sur le cancer 2008. Toronto, Ontario. http://www.cancer.ca/Canadawide/About\%20cancer/Cancer\%20statistics/Canadian\%20Cancer \%20Statistics.aspx?sc_lang=fr-ca

Spitzer, D., Neufeld, A., Harrison, M., Hughes, K., \& Stewart, M. (2003). Care giving in the transnational context: "My wings have been cut: where can I fly?" Gender and Society, 2(10), 1-20.

Statistique Canada. (p. de d.). Recensement de 2006-Population immigrante selon le lieu de naissance et la période d'immigration. Consulté à http://www40.statcan.gc.ca/102/cst01/demo24afra.htm

Tarlier, D.S. (2004). Beyond caring: The moral and ethical bases of responsive nurse-patient relationships. Nursing Philosophy, 5, 230-241.

Thorne, S.E, Bultz, B.D., \& Baile, W.F. (2005). Is there a cost to poor communication in cancer care?: A critical review of the literature. Psycho-Oncology, 14, 875-884.

Vydelingum, V. (2006). Nurses' experiences of caring for South Asian minority ethnic patients in a general hospital in England. Nursing Inquiry, 13, 23-32.

Widäng, F., Fridlund, B., \& Martensson, J. (2007). Women patients' conceptions of integrity within health care: A phenomenographic study. Journal of Advanced Nursing, 61, 540-548. 